\title{
Integration of post-mastectomy radiation and breast reconstruction*
}

\author{
J. L. Wright ${ }^{\mathrm{a}}$, P. G. Cordeiro ${ }^{\mathrm{b}}$, B. L. McCormick ${ }^{\mathrm{C}}$ \\ ${ }^{a}$ Department of Radiation Oncology, Memorial Sloan-Kettering Cancer Center, New York, NY, USA; \\ ${ }^{b}$ Department of Surgery, Plastic and Reconstructive Service, Memorial Sloan-Kettering Cancer Center, \\ New York, NY, USA; ' Department of Radiation Oncology, Memorial Sloan-Kettering Cancer Center, \\ New York, NY, USA.
}

\begin{abstract}
The following is a brief description of the reconstructive options available to patients undergoing mastectomy, and a review of the data available to guide clinicians who seek to safely integrate postmastectomy radiotherapy and breast reconstruction.
\end{abstract}

Keywords: Breast reconstruction; Post-mastectomy radiation

Post-mastectomy radiotherapy (PMRT) has traditionally been used to treat breast cancer patients with tumours larger than $5 \mathrm{~cm}$ or when four or more axillary lymph nodes are involved [1,2]. However, the use of PMRT is increasing as accumulating data suggest both a local control and survival advantage in patients with one to three positive axillary lymph nodes, as well [1,3-5]. Because the pathologic stage of disease is determined by definitive surgery, the role of PMRT is often not defined before surgery. The use of reconstruction in patients who receive PMRT is controversial; American Society of Clinical Oncology (ASCO) guidelines state that there is insufficient evidence to make recommendations or suggestions with regard to the integration of PMRT and reconstructive surgery [2].

*We have no sources of financial support to acknowledge.

Correspondence to: Beryl L. McCormick, MD, Memorial Sloan-Kettering Cancer Center, Department of Radiation Oncology, 1275 York Avenue, New York, NY 10021, USA. E-mail: mccormib@mskcc.org; Tel: +1 212 639 2950; Fax: +1212639 2417

Received: 01/08/07

Accepted: 18/08/07

BCO/644/2007/FO
The following is a brief description of the reconstructive options available to patients undergoing mastectomy, and a review of the data available to guide clinicians who seek to safely integrate PMRT and breast reconstruction.

There are two general categories of breast reconstruction techniques, autologous tissue reconstruction and expander-implant $(E-I)$ reconstruction. Autologous reconstruction entails reconstruction of the breast mound with autologous tissue from another site in the body. The most common autologous reconstruction is the transverse rectus abdominus myocutaneous (TRAM) flap, in which donor tissue from the lower abdomen including skin, subcutaneous fat and rectus abdominus muscle is used to reconstruct the breast mound. Other donor sites, including a latissimus dorsi musculocutaneous flap, or a gluteal flap, may be employed, depending on the patient's anatomy. Autologous reconstruction procedures are lengthy and can be associated with significant morbidity and prolonged healing period. This type of reconstruction may be contraindicated in the setting of comorbid conditions that impair wound healing, including obesity, diabetes, prior surgery, collagen vascular disease or an extensive smoking history. Autologous reconstruction is also 
subject to anatomical constraints, including the presence of an adequate donor site; thin women may lack sufficient tissue. In contrast, E-I reconstruction replicates the breast mound via placement of a permanent silicone or saline implant under the pectoralis muscle, and does not require donor tissue. This reconstruction is generally performed in two stages, with the initial placement of a tissue expander (TE), which is incrementally expanded with saline over a period of time, followed by exchange of the TE for a permanent implant. These procedures require significantly less operative time than autologous reconstruction. Both reconstructive techniques can be performed on an immediate basis, at the time of mastectomy, or a delayed basis, as a separate surgical procedure after healing from the mastectomy is complete.

There has been a great deal of controversy in the literature regarding the integration of PMRT and reconstruction. Two algorithms that incorporate both treatments have been published. One algorithm is delayed-immediate reconstruction [6], in which skin-sparing mastectomy with placement of a fully expanded TE is performed. After pathology is reviewed, a decision regarding the role of PMRT is made. In patients not requiring PMRT, reconstruction with autologous tissue or a permanent implant is performed shortly after mastectomy. If PMRT is indicated, the TE is deflated, radiation is delivered and the expander is later re-expanded. A final autologous reconstruction is then performed. Chemotherapy may be delivered in a neoadjuvant fashion, or between surgery and radiation. Another algorithm that has been reported involves definitive mastectomy with immediate TE placement, tissue expansion during adjuvant chemotherapy, exchange of TE for permanent implant, followed by radiation [7].

The three major criticisms of algorithms that include PMRT and reconstruction are the potential for increased complication rates and resultant inferior cosmetic outcomes, the possibility of compromised radiation design and the risk of inferior oncologic outcomes.

The early complications that have been reported in patients undergoing reconstruction and PRMT include fat or flap necrosis, vessel thrombosis, infection, haematoma, delayed wound healing, seroma, failed tissue expansion and expander deflation [7-11]. Late complications include pain, capsular contracture, implant deflation, leakage or exposure, and decrease in various measures of aesthetic outcome [7-11]. In general, the published series assessing complication rates and cosmetic outcomes are limited by small patient numbers and heterogeneous patient populations, as well as significant variation in the reported endpoints. There are no studies that we are aware of which prospectively compare E-I and autologous reconstruction. One study which retrospectively compares the two types of reconstruction reports a $53 \%$ incidence of complications within 2 years in patients treated with PMRT and E-I reconstruction vs. $12 \%$ if TRAM reconstruction is employed; patients received radiation either before or after reconstruction [10]. In contrast, another study reports no difference in major complications between TRAM and E-I reconstruction ( $0 \%$ vs. $5 \%$, not statistically significant), and an increased rate of minor complications in TRAM vs. E-I reconstruction (39\% vs. $14 \%)$; again patients received radiation either before or after reconstruction [8]. A comparison of patients who received immediate TRAM reconstruction followed by radiation vs. patients who received radiation followed by delayed TRAM reconstruction reported similar early complication rates, but decreased late complications with delayed reconstruction [11]. When E-I reconstruction is used, radiation usually occurs after reconstruction, as irradiated tissue is not amenable to expansion.

In general, an increased risk of complications and poorer cosmesis are consistently reported in patients who undergo any combination of breast reconstruction and radiation, compared to those who undergo reconstruction without radiation [12-15]. This is exemplified by a prospective study of 12 patients who underwent bilateral immediate E-I reconstruction and unilateral PMRT, which compares outcomes in the two breasts, using the non-irradiated breast as a control [13]. In this study, no discernible difference was identified between the two breasts in $40 \%$, grade of contracture was increased by one grade on the modified Baker scale in $50 \%$, and increased by two grades in $10 \%$. The delayed-immediate algorithm is reported to allow for superior cosmetic outcomes because re-expansion of the mastectomy skin provides additional breast skin to perform delayed reconstruction [16], though specific data on cosmetic outcomes have not been reported. The E-I algorithm has been reported to result in good-to-excellent aesthetic results in $80 \%$ of cases [7].

Based on the previously described literature, it is difficult to draw conclusions regarding the cosmetic superiority of any one form of reconstruction or any particular sequence of treatments, though initial tissue expansion after radiation is rarely feasible due to inelasticity of the tissue. Opinion on this topic can be sharply divided, and while some have concluded that PMRT and reconstruction cannot be safely integrated, many publications do support the feasibility of such a technique.

In addition to cosmetic concerns, some studies have raised the concern that irradiation of the 
immediately reconstructed breast results in inferior quality of radiation delivery $[17,18]$. These studies report that post-mastectomy radiation plans in patients with E-I or autologous reconstructions are often unsatisfactory in terms of providing broad coverage of the chest wall and internal mammary nodes (IMN), while adequately sparing the heart and lung. In contrast, another study reports adequate chest wall coverage and normal tissue sparing in patients treated with E-I reconstruction followed by intensity-modulated chest wall radiation whether or not IMN are treated, though normal tissue doses were higher if the IMN were targeted [19]. The delayed-immediate technique attempts to circumvent this problem by deflating the TE during radiation. This algorithm requires irradiation of the TE, and higher energy radiation is required to overcome interference of the magnetic component of the port with radiation delivery [20]. In general, adequate tissue sparing should be feasible if the IMN are not treated, and is more difficult to accomplish if they are. Treatment of the IMN is controversial and has not been shown to confer a benefit in survival or rate of distant metastasis [21,22].

Oncologic outcomes in patients undergoing PMRT and reconstruction have been reported to be acceptable [23-25]. However, a concern often raised is that delays in the initiation of radiation in the setting of immediate reconstruction could result in inferior disease control. Delay in the initiation of radiation therapy in the setting of adjuvant chemotherapy after mastectomy is generally considered acceptable; several studies report no increase in local failure rate if radiation is delayed until after adjuvant chemotherapy $[26,27]$. However, one meta-analysis showed a higher odds ratio for mortality if radiation was delayed by more than 6 months from the start of chemotherapy [28]. A delay of 8-12 weeks between surgery and the start of radiation is generally considered acceptable in breast-conserving therapy without chemotherapy [29-31].

Few studies looking at oncologic outcomes in patients undergoing reconstruction and PMRT assess treatment intervals. One study reports similar rates of local failure and distant metastasis in patients treated with PMRT with and without immediate implant reconstruction at a mean followup of 72 months, but treatment intervals were not reported [23]. Another study reports no significant difference in the incidence of local failure or distant metastasis in patients undergoing PMRT after immediate TRAM reconstruction compared to those who did not undergo reconstruction [24]. The interval from surgery to radiation in this study was similar whether or not reconstruction was performed, and was approximately 6 months.
Oncologic outcomes with the delayed-immediate technique have not been published, but should not be compromised as there is no delay between chemotherapy and radiation, due to the fact that the final reconstruction occurs after radiation. Using the E-I treatment algorithm, an 8-month interval between mastectomy and initiation of radiation, and an 8-week interval between chemotherapy and initiation of radiation have been reported, and local control, distant metastasis-free survival and overall survival at 5 years were $100 \%, 90 \%$ and $96 \%$, respectively [25].

The integration of breast reconstruction and PMRT remains controversial. Delayed-immediate reconstruction and $\mathrm{E}-\mathrm{I}$ reconstruction algorithms have been published, but there is no consensus on the best treatment approach. The most pertinent decisions for patients and clinicians are whether the possible complications and cosmetic outcomes are acceptable to a given patient, whether the IMN will need to be treated with radiation, and which treatment algorithm the treating physicians are most comfortable with. Decisions must be made on an individual basis, taking into account the patient's stage of disease, body habitus, comorbidities and expectations of the cosmetic outcomes.

\section{References}

1. Clarke M, Collins R, Darby S, et al. Effects of radiotherapy and of differences in the extent of surgery for early breast cancer on local recurrence and 15-year survival: an overview of the randomised trials. Lancet 2005; 366: 2087-2106.

2. Recht A, Edge SB, Solin LJ, et al. Postmastectomy radiotherapy: clinical practice guidelines of the American society of clinical oncology. J Clin Oncol 2001; 19: 1539-1569.

3. Overgaard M, Hansen PS, Overgaard J, et al. Postoperative radiotherapy in high-risk premenopausal women with breast cancer who receive adjuvant chemotherapy. Danish breast cancer cooperative group 82b trial. N Engl J Med 1997; 337: 949-955.

4. Overgaard M, Jensen MB, Overgaard J, et al. Postoperative radiotherapy in high-risk postmenopausal breast-cancer patients given adjuvant tamoxifen: Danish breast cancer cooperative group DBCG 82c randomised trial. Lancet 1999; 353: 1641-1648.

5. Ragaz J, Olivotto IA, Spinelli JJ, et al. Locoregional radiation therapy in patients with high-risk breast cancer receiving adjuvant chemotherapy: 20 -year results of the British Columbia randomized trial. J Natl Cancer Inst 2005; 97: 116-126.

6. Kronowitz SJ, Hunt KK, Kuerer HM, et al. Delayedimmediate breast reconstruction. Plast Reconstr Surg 2004; 113: 1617-1628.

7. Cordeiro PG, Pusic AL, Disa JJ, McCormick B, VanZee K. Irradiation after immediate tissue expander/implant 
breast reconstruction: outcomes, complications, aesthetic results, and satisfaction among 156 patients. Plast Reconstr Surg 2004; 113: 877-881.

8. Anderson PR, Hanlon AL, Fowble BL, McNeeley SW, Freedman GM. Low complication rates are achievable after postmastectomy breast reconstruction and radiation therapy. Int J Radiat Oncol Biol Phys 2004; 59: 1080-1087.

9. Vandeweyer E, Deraemaecker R. Radiation therapy after immediate breast reconstruction with implants. Plast Reconstr Surg 2000; 106: 56-60.

10. Chawla AK, Kachnic LA, Taghian AG, et al. Radiotherapy and breast reconstruction: complications and cosmesis with tram versus tissue expander/implant. Int $J$ Radiat Oncol Biol Phys 2002; 54: 520-526.

11. Tran NV, Chang DW, Gupta A, Kroll SS, Robb GL. Comparison of immediate and delayed free tram flap breast reconstruction in patients receiving postmastectomy radiation therapy. Plast Reconstr Surg 2001; 108: 78-82.

12. Krueger EA, Wilkins EG, Strawderman M, et al. Complications and patient satisfaction following expander/ implant breast reconstruction with and without radiotherapy. Int J Radiat Oncol Biol Phys 2001; 49: 713-721.

13. McCarthy CM, Pusic AL, Disa JJ, et al. Unilateral postoperative chest wall radiotherapy in bilateral tissue expander/implant reconstruction patients: a prospective outcomes analysis. Plast Reconstr Surg 2005; 116: 1642-1647.

14. Cordeiro PG, McCarthy CM. A single surgeon's 12-year experience with tissue expander/implant breast reconstruction: part I. A prospective analysis of early complications. Plast Reconstr Surg 2006; 118: 825-831.

15. Cordeiro PG, McCarthy CM. A single surgeon's 12-year experience with tissue expander/implant breast reconstruction: part II. An analysis of long-term complications, aesthetic outcomes, and patient satisfaction. Plast Reconstr Surg 2006; 118: 832-839.

16. Kronowitz SJ. Immediate versus delayed reconstruction. Clin Plast Surg 2007; 34: 39-50abstract vi.

17. Schechter NR, Strom EA, Perkins GH, et al. Immediate breast reconstruction can impact postmastectomy irradiation. Am J Clin Oncol 2005; 28: 485-494.

18. Motwani SB, Strom EA, Schechter NR, et al. The impact of immediate breast reconstruction on the technical delivery of postmastectomy radiotherapy. Int $\mathrm{J}$ Radiat Oncol Biol Phys 2006; 66: 76-82.

19. Koutcher LBA, Cordeiro PG, McCormick BL, Hunt M, Beal K. Postmastectomy intensity modulated radiation therapy (IMRT) in women who undergo immediate breast reconstruction.
20. Damast S, Beal K, Ballangrud A, et al. Do metallic ports in tissue expanders affect postmastectomy radiation delivery? Int J Radiat Oncol Biol Phys 2006; 66: 305-310.

21. Fowble B, Hanlon A, Freedman G, et al. Internal mammary node irradiation neither decreases distant metastases nor improves survival in stage I and II breast cancer. Int J Radiat Oncol Biol Phys 2000; 47: 883-894.

22. Freedman GM, Fowble BL, Nicolaou N, et al. Should internal mammary lymph nodes in breast cancer be a target for the radiation oncologist? Int $J$ Radiat Oncol Biol Phys 2000; 46: 805-814.

23. Vandeweyer E, Hertens D, Nogaret JM, Deraemaecker R. Immediate breast reconstruction with saline-filled implants: no interference with the oncologic outcome? Plast Reconstr Surg 2001; 107: 1409-1412.

24. Huang CJ, Hou MF, Lin SD, et al. Comparison of local recurrence and distant metastases between breast cancer patients after postmastectomy radiotherapy with and without immediate tram flap reconstruction. Plast Reconstr Surg 2006; 118: 1079-1086.

25. Wright JL, Cordeiro PG, Ben-Porat L, et al. Mastectomy with immediate expander-implant reconstruction, adjuvant chemotherapy and radiation for stage II-III breast cancer: treatment intervals and clinical outcomes. Int $J$ Radiat Oncol Biol Phys 2007 September 11 [Epub ahead of print].

26. Sartor Cl, Peterson BL, Woolf S, et al. Effect of addition of adjuvant paclitaxel on radiotherapy delivery and locoregional control of node-positive breast cancer: cancer and leukemia group b 9344. J Clin Oncol 2005; 23: 30-40.

27. Metz JM, Schultz DJ, Fox K, et al. Analysis of outcomes for high-risk breast cancer based on interval from surgery to postmastectomy radiation therapy. Cancer $J$ 2000; 6: 324-330.

28. Whelan TJ, Julian J, Wright J, Jadad AR, Levine ML. Does locoregional radiation therapy improve survival in breast cancer? A meta-analysis. J Clin Oncol 2000; 18: 1220-1229.

29. Nixon AJ, Recht A, Neuberg D, et al. The relation between the surgery-radiotherapy interval and treatment outcome in patients treated with breast-conserving surgery and radiation therapy without systemic therapy. Int J Radiat Oncol Biol Phys 1994; 30: 17-21.

30. Vujovic O, Perera F, Dar AR, et al. Does delay in breast irradiation following conservative breast surgery in node-negative breast cancer patients have an impact on risk of recurrence? Int J Radiat Oncol Biol Phys 1998; 40: 869-874.

31. Froud PJ, Mates D, Jackson JS, et al. Effect of time interval between breast-conserving surgery and radiation therapy on ipsilateral breast recurrence. Int $J$ Radiat Oncol Biol Phys 2000; 46: 363-372. 